\title{
Functional trait changes in the floras of 11 cities across the globe in response to urbanization
}

\author{
Estibaliz Palma, Jane A. Catford, Richard T. Corlett, Richard P. Duncan, Amy K. Hahs, \\ Michael A. McCarthy, Mark J. McDonnell, Ken Thompson, Nicholas S. G. Williams \\ and Peter A. Vesk
}

E.Palma (epalma@student.unimelb.edu.au), J. A. Catford, M. A. McCarthy, A. K. Hahs, M. J. McDonnell and P. A. Vesk, School of BioSciences, The Univ. of Melbourne, VIC, Australia. JAC also at: Fenner School of Environment and Society, The Australian National Univ., Canberra, ACT, Australia, and Dept of Ecology, Evolution and Behavior, Univ. of Minnesota, Saint Paul, MN, USA, present address: Biological Sciences, Univ. of Southampton, Southampton, UK. - R. T. Corlett, Center for Integrative Conservation, Xishuangbanna Tropical Botanical Gardens, Chinese Academy of Sciences, Menglun, Yunnan, China. - R. P. Duncan, Inst. for Applied Ecology, Univ. of Canberra, ACT, Australia. K. Thompson, Dept of Animal and Plant Sciences, Univ. of Sheffield, Sheffield, UK. - N. S. G. Williams, School of Ecosystem and Forest Sciences, The Univ. of Melbourne, VIC, Australia. AKH, MJM and NSGW also at: Australian Research Centre for Urban Ecology, Royal Botanical Gardens Victoria, VIC, Australia.

\begin{abstract}
Urbanization causes major environmental changes globally, which can potentially homogenize biota across cities through the loss and gain of particular types of species. We examine whether urban environments consistently select for plants with particular traits and the implications of such changes on the functional composition of urban floras. We classified plant recorded in 11 cities around the globe as species that have either colonized (arrived and naturalized), persisted or been lost (local extirpation) following urbanization. We analyzed how 10 traits previously linked with plant responses to environmental conditions explained membership of these three groups, by comparing colonisers with persistent and extirpated plants through individual city-level Bayesian models. Then, we used meta-analysis to assess consistency of traits across urban areas. Finally, we explored several possible scenarios of functional change using these results.

On average, urban colonizers had heavier seeds, unspecialised nutrient requirements, were taller and were annual species more often, especially when compared to locally extirpated plants. Common trends of functional change in urban plant communities include shifts towards taller and heavier-seeded plants, and an increased prevalence of the short-lived species, and plants without mutualistic nutritional strategies. Our results suggest that plant traits influence the species that succeed in urban environments worldwide. Different species use different ecological strategies to live in urban environments, as suggested by the importance of several traits that may appear as trait constellations. Plant height and seed mass were the only traits associated with both colonizer and extirpated plant status in urban environments. Based on our data, predicting colonization in urban environments may be easier than identifying extirpation-prone plants; albeit some regional variation, colonization seems strongly driven by environmental conditions common to most cities (e.g. altered disturbance regimes), whereas extirpation may depend more on processes that vary across cities.
\end{abstract}

Human-driven ecosystem degradation and habitat loss alter species distributions worldwide (Vitousek et al. 1997). These processes are particularly intense in urban areas, where environments are heavily modified to suit human needs and desires. Cities share common environmental features regardless of their geographical location (including microclimates, high levels of disturbance and fragmentation, elevated concentration of nitrogen in soils, and water stress; Grimm et al. (2008)) and can thus exert similar pressures on biological communities. As a consequence, urban environments around the world are expected to favour species with similar biological and ecological characteristics that allow them to persist in these highly modified habitats (McKinney and Lockwood 1999).
According to ecological filtering theory (Keddy 1992), only some species will persist in a given area following environmental change (Williams et al. 2009). Urbanization is a strong driver of environmental change, and plant species respond to it in different ways (Olden and Poff 2003). Depending on the ability of plants to cope with the altered conditions, some species will become locally extirpated while others will persist. Urbanization will also offer opportunities for new plants, not previously part of the local flora, to join the community through changes in local ecological conditions, like increases in resource availability through eutrophication (Shochat et al. 2006, Grimm et al. 2008, McDonnell and Hahs 2015). Plant responses to urbanization-driven changes depend on individual species traits (McGill et al. 
2006, Lavorel et al. 2007). For example, Williams et al. (2015) found that seed mass, height and annual life-history increase in response to urban stressors, McKinney (2002) concluded that wind dispersal is a common attribute of plants adapted to urban disturbance, and Duncan et al. (2011) determined that short-stature, small-seeded plants are more likely to go extirpated in urban areas. Comparison of traits among species that respond differently to urbanization-driven environmental change can increase understanding of changes in species distribution patterns (Díaz et al. 2004, Olden and Rooney 2006). Traits consistently shared by plants that have been lost from, have persisted in, or have entered urban plant communities may point to the ecological strategies that species use to cope with environmental constraints and the mechanisms underlying responses to urbanization. A simultaneous assessment of extirpated, persistent and colonizing species untangles changes that occur exclusively due to environmental change, buffering issues related to introduction bias - a challenging problem for trait-oriented colonization studies (Colautti et al. 2006). Such an approach also assists in creating a clear picture of the changes that occur and their consequences for the flora composition.

By selecting for species with certain traits, urbanization can result in biological homogenization where communities lose their distinctiveness and become more genetically, taxonomically or functionally similar (Olden and Rooney 2006). The taxonomic composition of urban floras is known to be slowly converging worldwide (La Sorte et al. 2014), but the magnitude and nature of changes in the functional composition of urban floras is still unclear (Olden 2006). We expect that urbanization will alter the functional composition of local plant communities in a consistent manner across cities, with successful plants being those able to deal with highly disturbed environments (Williams et al. 2009, Thompson and Davis 2011). However, functional homogenization is multifaceted; the floristic similarity among cities around the world will depend on the original local flora, the strength of different urban stressors and the functional characteristics of both resident and newly arriving plants (Olden and Poff 2003). Depending on these elements, urbanization-caused plant extirpation and colonization will drive different scenarios of functional composition change (Fig. 1). In this paper, we use the term colonization (or colonizers) to refer to new plants that successfully enter and establish in the community. We use colonization in the broadest sense of the word, and do not consider species' biogeographic origin or their abundance, geographic extent or impact within cities. Colonizers may have similar characteristics to plants that have been able to persist under altered environmental conditions (Fig. 1a); this may partially result from non-random human-driven habitat transformation within cities, leading to the disappearance of species with particular traits, and would represent a narrowing of community functional composition. If colonizers show more extreme values for characteristics that promote persistence (Fig. 1b), it would suggest that there has been a shift in the types of species that succeed in cities (McKinney and Lockwood 1999), which would strongly point to habitat filtering as the main driver of functional change. Alternatively, traits of colonizers may be more similar to those of extirpated plants than to those of persisting plants (Fig. 1c), potentially resulting in no change to community functional composition. This would occur if colonizers displace the (now extirpated) plants or due to direct human-driven replacement. Finally, persistent and extirpated plants may share similar characteristics to each other, but colonizers differ (Fig. 1d); this scenario could result from preferential introduction of certain (a)

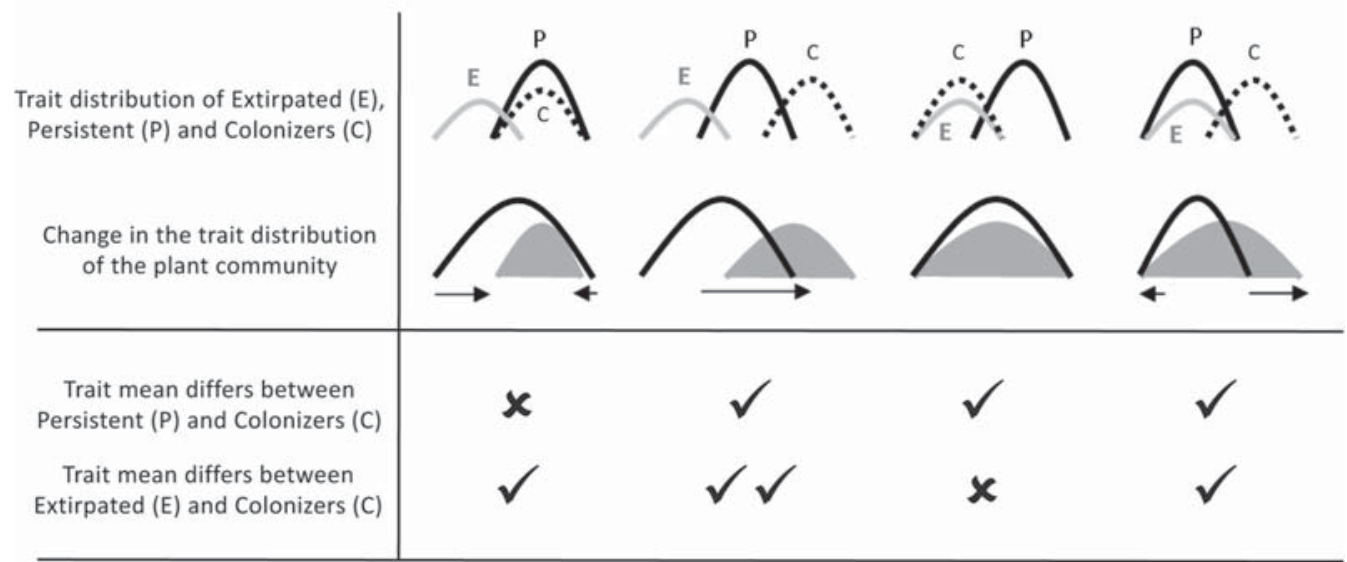

Figure 1. Possible scenarios of functional change after extirpation and colonization occur. (a) Colonizers are functionally similar to persistent plants: community trait distribution narrows. (b) Colonizers and extirpated plants occupy opposite extremes of the trait gradient: community trait distribution shifts. (c) Colonizers are functionally similar to extirpated plants: community trait distribution does not change. (d) Colonizers are functionally different from extirpated and persistent plants (which are functionally similar to each other); community trait distribution widens. First row represents the trait distribution for extirpated (E, grey lines), persistent (P, black lines) and colonizer (C, dashed lines) plants. Second row includes the trait distribution of the plant community before (black line) and after (grey shadow) extirpation and colonization occur for each scenario. Third and fourth lines represent the particular combination of meta-analysis results that points to a given scenario; ticks symbolize a consistent difference between plant groups for a given trait across cities; crosses indicate the lack of such a pattern. In scenario (b) the difference between colonizers and persistent plants is smaller than the difference between colonizers and extirpated plants, whereas in scenario (d) both differences are of similar magnitude. 
types of species by humans (i.e. introduction bias) and would indicate that the functional composition of the plant community has widened.

Using data from 11 cities in North America, Oceania and Asia, we investigate whether urban environments consistently select for particular types of plants by comparing the traits of species that have colonized to those that have persisted (to test niche overlap) or become extirpated (to test functional replacement) in urban environments. Based on these results, we reconcile the four scenarios of functional change (Fig. 1) by determining whether community-level trait distributions in these 11 cities are shrinking, shifting, remaining similar or expanding. We use data from the same cities as Duncan et al. (2011) who compared the traits of locally extirpated and extant native plant species. Although Duncan et al. (2011) were able to characterise species that are typically lost from urban environments from the original species pool, they were unable to show whether these losses were part of a systematic functional change at the community level, as the comparisons with colonizers were not investigated (Fig. 1). Our approach examines the characteristics of species that are both lost and gained from urban environments, which is critical for ascertaining whether urbanization is selecting for functionally similar floras around the world. It allows us to infer whether particular traits are similarly (but oppositely) involved in extirpation and colonization, whether they are involved in colonization or extirpation only, or whether colonizers are replacing functionally similar extirpated urban plants. This work offers the opportunity to improve understanding of how assembly rules of plant communities have changed through urbanization and represents a step forward in the study of functional homogenization in human-dominated habitats. Given the growing rates of urbanization around the world (Cohen 2003), this work has the potential to improve the knowledge of processes that are expected to become increasingly prevalent.

\section{Methods}

\section{Cities and urban flora}

This study includes plant records from 11 cities in North America (Chicago, Los Angeles, New York, San Diego, San Francisco and Worcester), Oceania (Adelaide, Auckland and Melbourne) and Asia (Hong Kong and Singapore). All of them were founded after $1800 \mathrm{AD}$, with the exception of New York (1624 AD) and Worcester (1722 AD) (see Duncan et al. (2011) for more details). Although the size of the cities and the proportion of their areas surveyed differ, every city is represented by a gradient of urban development, from highly urbanized core areas to semi-urban environments. Over the last few centuries, urbanization has altered the composition of flora that was historically associated with these regions. We collected information about the floristic composition of the regions, before and after city establishment, from peer-reviewed papers, unpublished herbarium reports and agency databases. The original sources showed variation in the methods used to define the city boundary, with some closely aligned with the extent of the built-up area and others including nearby natural areas. For each city, plant species were classified into one of the following categories: persistent - recorded in initial surveys shortly after city establishment and still present today; extirpated - recorded in initial surveys shortly after city establishment but currently determined locally extirpated by the study authors; or colonizer - absent in initial surveys of the local area under consideration for each city but recorded in subsequent surveys after city establishment. As far as it was possible, the last category only included plants able to produce reproductive offspring (naturalized, sensu Richardson et al. (2000)), excluding plants whose populations can only be maintained through cultivation.

The dataset included a total of 10258 species with 1711 extirpated species, 7091 persistent species and 2353 colonizer species recorded in one or more cities (Fig. 2a). The total number of species varied markedly among cities, ranging from 869 records in Los Angeles to 2402 in Singapore. The proportion of species in each group - extirpated, persistent or colonizer - also varied greatly among cities (Fig. 2b). Of the total species recorded at city-level, colonizers contributed between 9\% (in Singapore) to 74\% (in Auckland) of the records, and extirpated plants contributed from 1\% (in San Diego) to 25\% (in Singapore). We followed the Angiosperm Phylogeny Group (Angiosperm Phylogeny Group 2003) for taxonomy, and merged subspecies and varieties to species level.

\section{Trait information}

We selected 10 plant traits thought to relate to plant extirpation risk, colonization success and response to urbanization (see Table 1 for references): dispersal syndrome, growth form, longevity, clonality, nutrient uptake strategy,

\section{(a)}

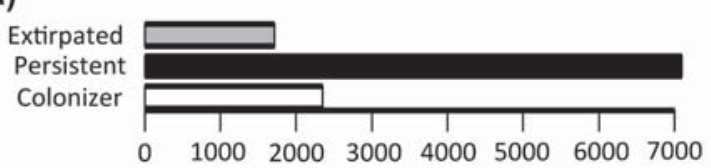

(b)

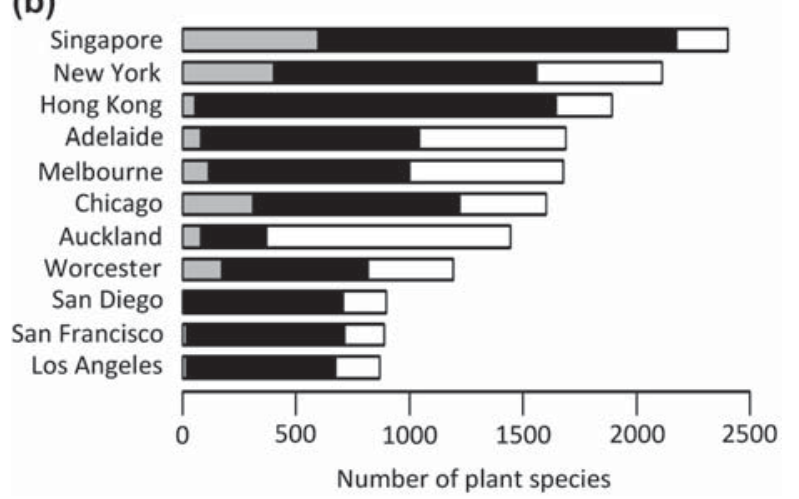

Figure 2. Number of plants recorded as extirpated (grey), persistent (black) and colonizer (white) for (a) all cities combined and (b) each city. Some species belong to different categories in different cities. 
photosynthetic pathway, spinescence, pollination syndrome, plant height and seed mass. We compiled information for each species from published works, trait databases, and expert knowledge. Pollination syndrome was defined as abiotic or biotic. The rest of the traits were initially categorised following protocols formalised by Cornelissen et al. (2003) and subsequently grouped into broader categories to meet analytical requirements. Table 1 includes categories of qualitative traits and, if any, the assumptions made when assigning specific categories or values to species.

\section{Data analysis}

This paper is divided in two sections. First, we identified whether urban environments consistently select for and against particular traits by comparing the traits of colonizer, persistent and extirpated plants. Then, we used our results to explore possible scenarios of functional change within urban plant communities, as shown in Fig. 1.

\section{Traits associated with colonizer, persistent and extirpated plants in urban environments}

We used the same two-step modelling approach - based on Duncan et al. (2011) - to compare colonizers with persistent plants and colonizers with extirpated plants. The former comparison is based on 9101 species and 14800 records; the latter comparison is based on 4020 species and 6588 records. More details about the datasets can be found in the Supplementary material Appendix 1, Table A3. For each comparison, we first conducted independent analyses for each of the 11 cities, and then used meta-analysis to combine and summarize the results at city-level and assess consistency of the observed patterns.

1) Independent analysis of each city. We assessed whether traits of colonizers differ from 1) persistent or 2) extirpated plants using hierarchical logistic regressions of the form:

$\mathrm{Y}_{\mathrm{i}} \sim$ Bernoulli $\left(\mathrm{p}_{\mathrm{i}}\right)$

$\operatorname{logit}\left(\mathrm{p}_{\mathrm{i}}\right)=\alpha+\Sigma\left(\beta_{\mathrm{k}} * \mathrm{X}_{\mathrm{ik}}\right)+\gamma_{\mathrm{f}}$

where $Y_{i}$ is the observed binary state of urban species, being either colonizer or persistent/extirpated. The probability of being a colonizer $\left(\mathrm{p}_{\mathrm{i}}\right)$ is modelled as a linear logistic function, with intercept $\alpha$, regression coefficients $\beta$ for traits X, and plant family effects $\gamma$. The intercept $\alpha$ represents the estimated probability of being a colonizer for species belonging to the reference class of all categorical traits and having average values of height and seed mass (Table 1). $\mathrm{X}_{\mathrm{ik}}$ refers to the value of explanatory trait $k$ for species $i$. Continuous traits were log-transformed and standardized to a standard deviation of 0.5 (Gelman and Hill 2007). Multicategorical traits with $n$ categories were coded as $n-1$ binary traits (dummy variables) and compared to the reference category (Table 1 ). The regression coefficient $\beta_{k}$ stands for the estimated effect of trait $k$ on the probability of a species being a colonizer. Traits with $\beta$ values different from zero point to a correlation between colonizers and the trait and, therefore, these traits are useful to distinguish them from persistent/extirpated plants. The term $\gamma_{\mathrm{f}}$ stands for the effect of plant family $f$ on the probability of being a colonizer and accounts for taxonomic relatedness. The 
estimated effects of traits and family on the probability of being a colonizer are conditional on the other explanatory variables, meaning that each of them accounts for the effect of the rest. Further details of the statistical models and the code used for modelling can be found in the Supplementary material Appendix 1 and 3.

2) Meta-analysis across cities. Meta-analysis comprises a set of tools that combine the magnitude or effect of several outcomes from primary studies addressing the same research question with different datasets but a comparable scale (Koricheva et al. 2013). These statistical methods weight the varying precision of effect estimates, therefore accounting for the unequal statistical power of studies and improving control of type II error rates (Arnqvist and Wooster 1995).

Using results from the analysis of individual cities, we modelled the effect of the previously assessed ten traits on the probability of species being colonizers as drawn from a normal distribution with a common mean:

$\beta_{\mathrm{jk}} \sim \operatorname{Normal}\left(\mathrm{B}_{\mathrm{k}}, \operatorname{sqrt}\left(\sigma_{\beta \mathrm{k}}^{2}+\sigma_{\mathrm{j} \beta \mathrm{k}}^{2}\right)\right)$

where $\mathrm{B}_{\mathrm{k}}$ is the average effect of trait $k$ on colonizer prevalence within urban environments. The standard deviation includes two parameters: $\sigma_{\beta \mathrm{k}}$ and $\sigma_{i \beta \mathrm{k}}$, which represent the variation of the effect $\beta$ of trait $k$ among cities, and its associated uncertainty for each city $j$, respectively. City was treated as a random effect. The meta-analysis dealing with colonizer and persistent plants used the estimates yielded from all the eleven previous city-level models; the metaanalysis dealing with colonizers and extirpated plants used the estimates yielded from eight of the previous city-level models (San Diego, San Francisco and Los Angeles were excluded due to convergence issues; Supplementary material Appendix 1). Effects of traits were considered consistent when their 95\% credible intervals did not overlap zero.

Given that cities included in this work were not randomly selected across the globe, we explored whether the effect of traits on extirpation and colonization patterns were more similar among cities belonging to the same biogeographic region than from other regions. To do so, we ran the same meta-analyses, this time including a random effect accounting for the fact that cities belong to one of the following biogeographic regions: east coast of the United States, west coast of the United States, Asia, and Oceania. Given the limited number of cities and biogeographic regions in our dataset, the trends found by this approach are highly uncertain, and we do not present the estimated effects of traits under this analytical scenario in the main body of the paper (but see Supplementary material Appendix 1). However, this assessment does suggest that factors that vary at the region-level mediate the way in which plant traits affect species' responses to urbanization.

\section{Exploration of plant community functional change in urban environments}

We explored changes in the functional composition of urban floras after species extirpation and colonization by simultaneously inspecting the model coefficients estimated from the previous two meta-analyses. Plotting the results of both comparisons (colonizers versus persistent, and colonizers versus extirpated plants) allowed us to ascertain the types of changes that have occurred in the functional composition of the 11 urban floras (Fig. 1). If we found consistent positive or negative effects of traits only for one of the comparisons, we can infer that the trait distribution at community-level is shrinking (Fig. 1a) or has not changed (Fig. 1c). If we find consistent effects of traits for both comparisons, we can infer that the community-level trait distribution is shifting (Fig. 1b; if the effect between colonizers and extirpated plants is larger than the effect between colonizers and persistent plants) or is becoming wider (Fig. 1d; if both effects show similar magnitude).

\section{Results}

\section{Do traits of colonizing and persistent plants differ?}

Several traits appeared to influence the presence of colonizers versus persistent plants across urban areas (Fig. 3a and 4a). Taller and heavy-seeded species were more likely to be colonizers, as indicated by positive effect sizes where $95 \%$ confidence intervals do not overlap zero. The city-level median height for colonizers and persistent plants was around $0.85 \mathrm{~m}$, while it was $0.5 \mathrm{~m}$ for extirpated plants; the city-level median seed mass for colonizers, persistent and extirpated groups was $1.41,0.82$ and $0.55 \mathrm{~g}$, respectively. Annuals, spiny plants and species that rely on biotic pollination were also more likely to be colonizers (i.e. perennials, non-spiny plants and species that rely on abiotic pollination were more likely to be persistent plants). Despite large uncertainty, plants with specific strategies of nutrient acquisition (nitrogen fixation, mycorrhizal associations or other strategies) were underrepresented among colonizers. Woody plants were the only growth form underrepresented among colonizers, although the effect shows some uncertainty, which may point to variation among cities. Dispersal strategies based on wind and external seed attachment on animals, and clonal reproduction based on rhizomes (but not aboveground organs) also reduced the presence of colonizers in urban areas. We found no consistent effect of the remaining traits or trait categories on the presence of colonizers and persistent plants across urban areas. Refer to the Supplementary material Appendix 1, for results at city-level.

\section{Do traits of colonizing and extirpated plants differ?}

In general, the patterns found in the previous comparison held for the comparison between colonizers and extirpated plants, although the uncertainty for the latter estimates was generally greater (Fig. 3b and 4b; city-level results in Supplementary material Appendix 1). The effects of most traits were similar in direction and magnitude: taller, heavy-seeded and annual plants were consistently more likely to be colonizers across urban areas. Equally, spiny species and plants relying on biotic pollination were more likely to be colonizers. Wind-dispersed species, and plants with nitrogen fixing ability, mycorrhizal associations or other strategy for nutrient uptake were more frequently found among extirpated plants. No consistent effect was found for the remaining traits.

The effect of traits on colonization compared with both persistence and extirpation in urban floras depends on 


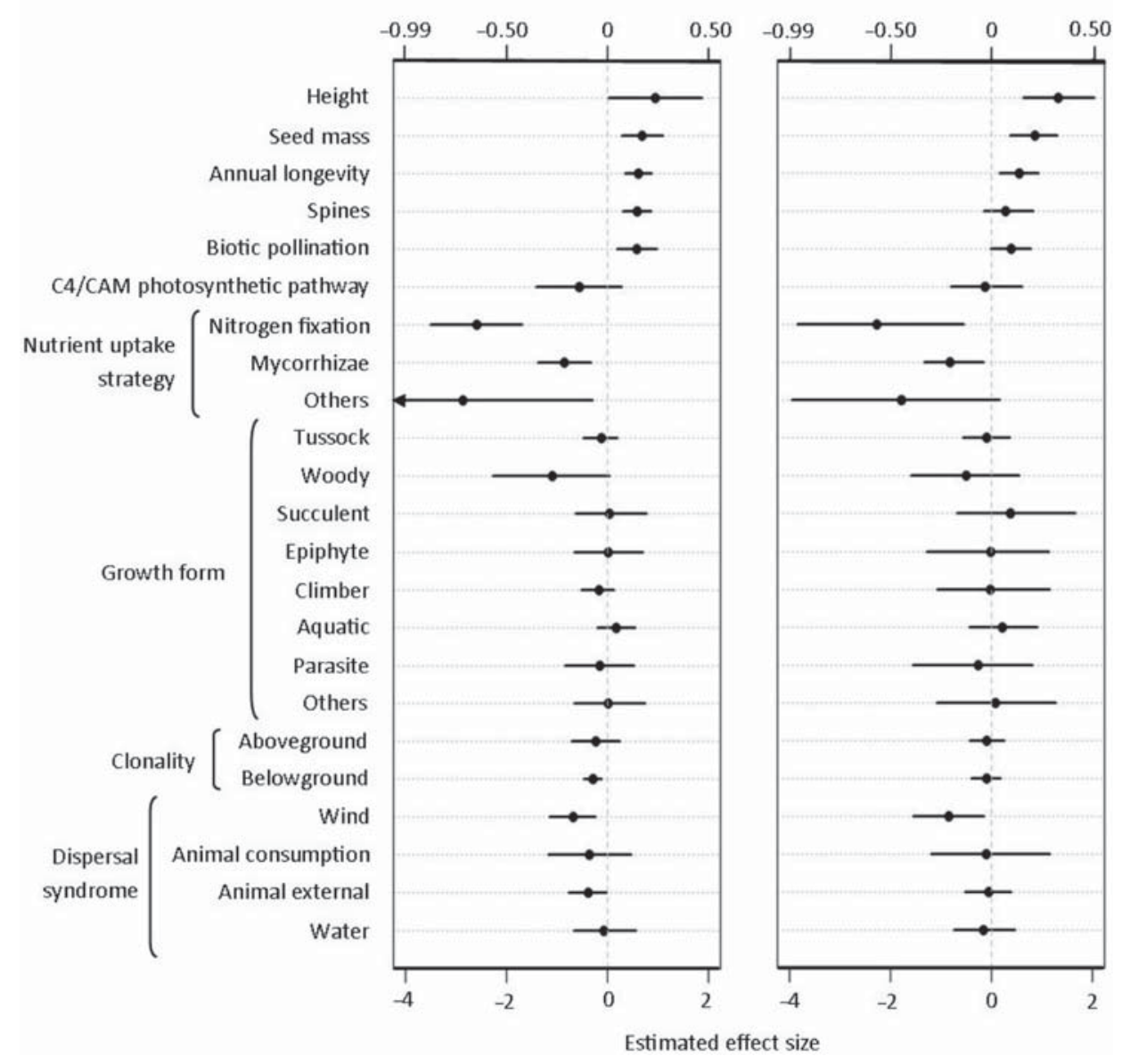

Figure 3. Estimated global effect of traits on the prevalence of colonizers over (a) persistent across 11 cities and (b) extirpated urban plants across 8 cities. Black dots represent mean effects and lines represent $95 \%$ credible intervals. Large positive and negative effects indicate traits that strongly characterise colonizers compared to persistent or extirpated plants. The $\mathrm{x}$ axis scale in the bottom represents the effect of the traits on the log odds of colonizers over persistent/extirpated plants: an effect of one means that colonizers are 2.7 times more likely to occur than the other group if they have that particular trait. The lower limit of the credible interval for the effect of 'Nutrient uptake strategy: others' in panel (a) is -7.2. The upper $\mathrm{x}$ axis represents the maximum change in the probability of being an colonizer given a change of one unit in the trait under consideration (for continuous traits) or belonging to the category under consideration compared to the reference category (for categorical traits), assuming that species with average traits, or belonging to the reference category, have the same probability of being colonizer or persistent/extirpated.

city-level specific processes, as well as regional biogeographic features (Fig. 5).

\section{How do colonization and extirpation influence the trait distribution of urban plant communities?}

Figure 6 shows the results from both meta-analyses simultaneously. We found that high values of height and seed mass are positively associated with both persistence and colonization, and negatively associated with extirpation (Fig. 6b), pointing to a shift in the functional composition of urban communities towards taller and heavier-seeded plants. Annual life-history, spinescence and biotic pollination are positively associated with colonization, whereas wind dispersal and nutrient uptake strategies such as nitrogen fixation and mycorrhizae are negatively associated with it (Fig. 6d). However, none of these traits affect the probability of local extirpation. This means that, after extirpation and colonization, the urban community composition has broadened to include more plants with annual longevity, spines, unspecialised dispersal and nutrient requirements, and relying on biotic pollination. Succulent plants tend to persist in urban environments (Fig. 6a), but they do not show a higher proportion among colonizers compared to the reference growth form (i.e. herbs). For plants that are clonal, animal dispersed and rely on C4 or CAM photosynthetic pathways, we found that colonizers replaced functionally similar extirpated plants (Fig. 6c); therefore, functional space characterized by these traits remains unchanged before and after urbanization. Remaining trait categories showed no consistent effects on colonization or extirpation patterns.

\section{Discussion}

Our results suggest that plant traits influence the types of species that fail and succeed in urban environments around 
(a)
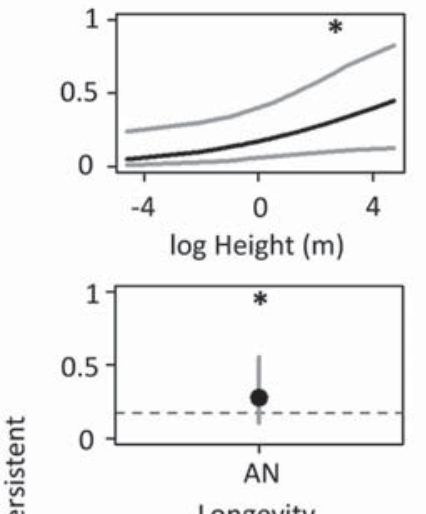

ngevity

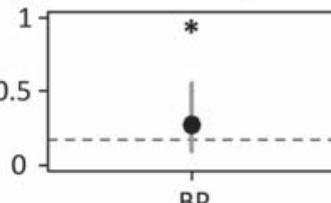

Pollination

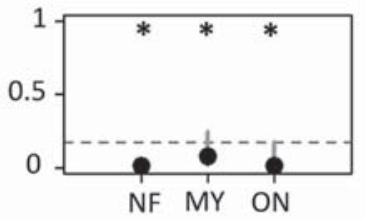

Nutrient uptake strategy

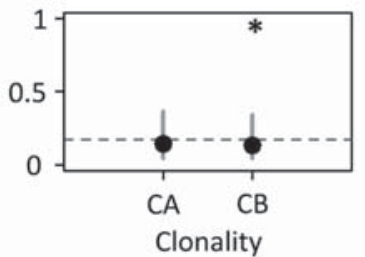

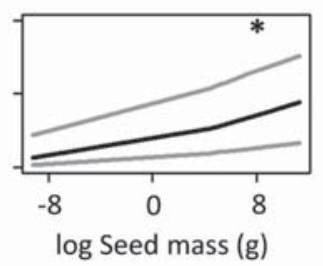

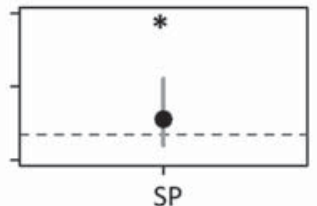

Spinescence

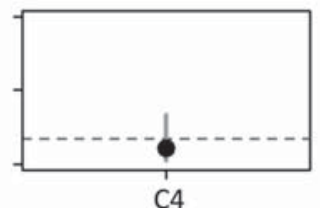

Photosynthetic pathway

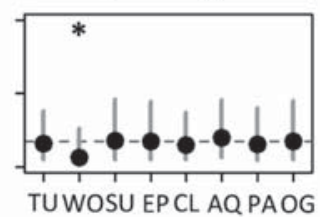

Growth form

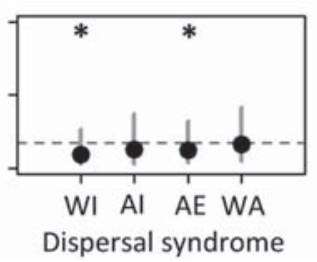

(b)

Colonizer vs Extirpated
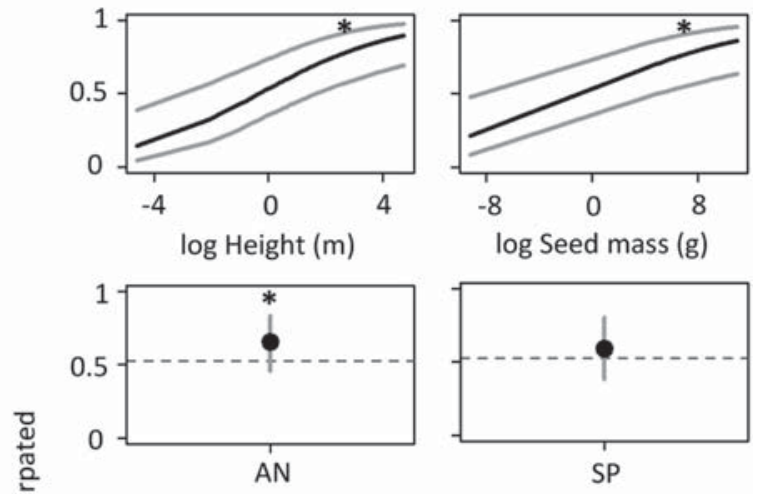

Spinescence

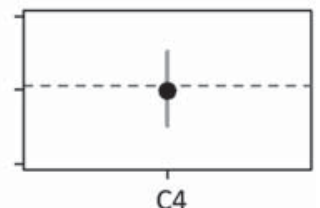

Photosynthetic pathway

Pollination

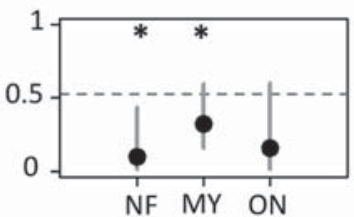

Nutrient uptake strategy

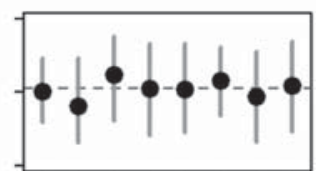

TUWOSU EPCL AQ PAOG Growth form
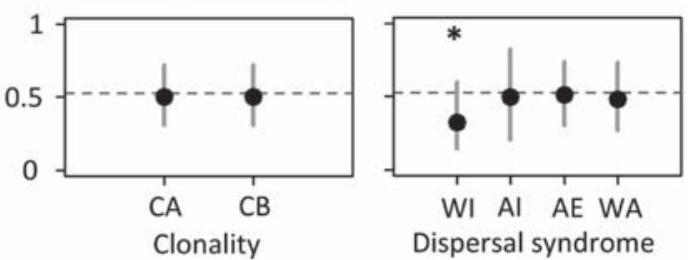

Figure 4. Predicted prevalence of colonizers over (a) persistent and (b) extirpated in urban plants across trait values or categories, based on estimated effects of traits for the meta-analysis. Black dots and lines represent mean effects and grey lines represent $95 \%$ credible intervals. Horizontal grey dashed lines represent the estimated ratio of colonizers and persistent/extirpated for plants belonging to the reference category of categorical traits. Dots above the horizontal line mean an increase in the proportion of colonizers, compared to the reference category; dots below the horizontal line mean an increase in the proportion of persistent (a) or extirpated (b) plants, compared to the reference category. Plots are based on eleven (a) and eight cities (b). ${ }^{*}$ Indicates traits with consistent effects. Codes for categorical traits: $\mathrm{AN}=$ annual longevity, $\mathrm{SP}=$ spines, $\mathrm{BP}=$ biotic pollination, $\mathrm{C} 4=\mathrm{C} 4$ or $\mathrm{CAM}$ photosynthetic pathway, $\mathrm{NF}=$ nitrogen fixer, $\mathrm{MY}=$ mycorrhizae, $\mathrm{ON}=$ other nutrient uptake strategy, $\mathrm{TU}=$ tussock, $\mathrm{WO}=$ woody, $\mathrm{SU}=$ succulent, $\mathrm{EP}=$ epiphyte, $\mathrm{CL}=$ climber, $\mathrm{AQ}=$ aquatic, $\mathrm{PA}=$ parasite, $\mathrm{OG}=$ other growth form, $\mathrm{CA}=$ clonal aboveground, $\mathrm{CB}=$ clonal belowground, $\mathrm{WI}=$ wind dispersal, $\mathrm{AI}=$ animal dispersal (consumption), $\mathrm{AE}=$ animal dispersal (external), WA = water dispersal.

the world. Some of the traits we investigated (plant height and seed mass) were associated with both plant extirpation and colonization processes in urban environments. Other traits appeared to relate to only one of these processes: for example, plant longevity and nutrient acquisition strategy appeared to be associated with colonization but unrelated to extirpation. The varying influence of different plant traits on extirpation and colonization (Fig. 6) leads to a variety of functional changes in the flora (e.g. shift towards novel trait values, shrinking of existing trait distributions) in response to urbanization. Our results indicate that plants in urban environments can follow different pathways to persist in the local flora (Hodkinson and Thompson 1997). For the limited number of traits and cities included in this work, more traits were found to be related to colonization than extirpation in urban areas (Duncan et al. 2011). In line with findings by Jeschke and Strayer (2008), our results suggests that colonization is a stronger force than extirpation in altering the functional composition of urban floras in a consistent manner across the urban areas under investigation, and that explaining and predicting why certain plants colonize urban environments, more generally, may be easier than explaining why certain plants become extirpated. Extirpation may be more strongly driven by local processes that vary across cities, whereas colonization may be strongly related to characteristics shared by many cities around the world, such as 
(a)

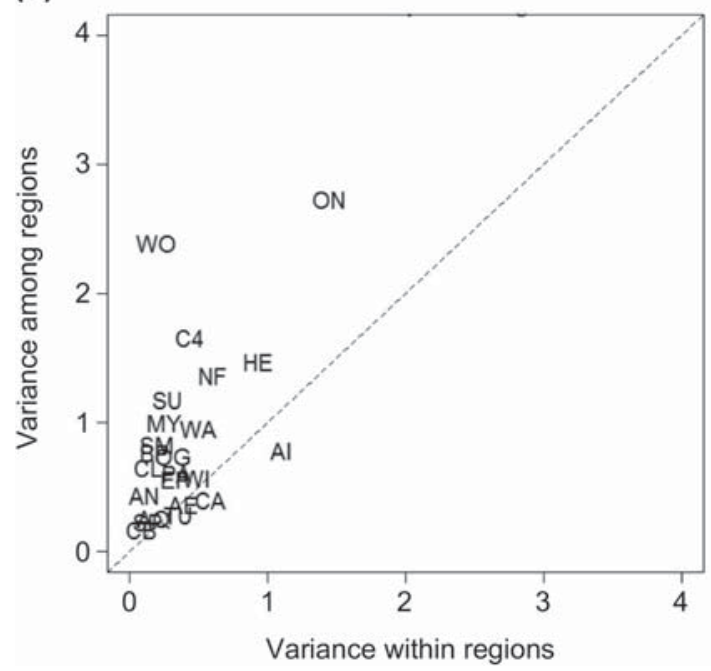

(b)

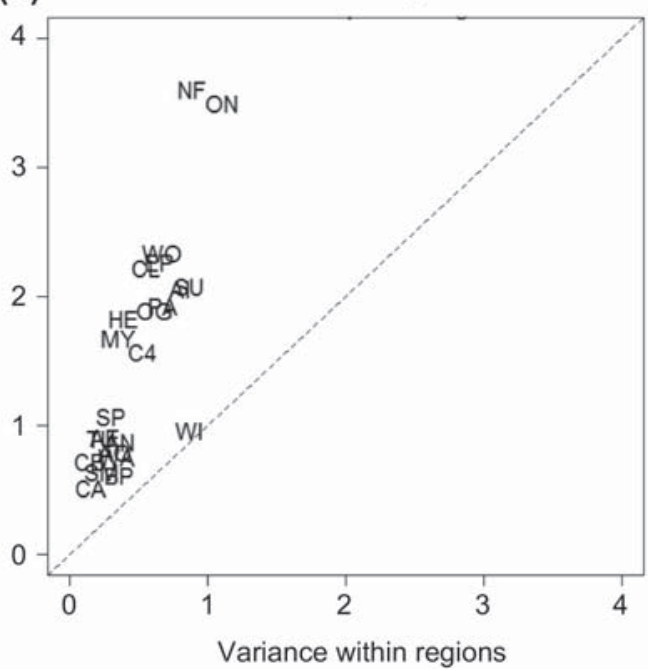

Figure 5. Partitioning of variance sources in the effect of traits on the log odds of colonizers over (a) persistent and (b) extirpated plants. Variability due to biogeographic region is shown in the y axis; variability due to individual city features within biogeographic regions is shown in the $\mathrm{x}$ axis. The effect of traits above the dashed line show most of their variation at regional level. Traits below the dashed line show more variation among cities within regions than among regions. Codes for traits: $\mathrm{SM}=$ seed mass, $\mathrm{HE}=$ height, $\mathrm{AN}=$ annual longevity, $\mathrm{SP}=$ spines, $\mathrm{BP}=$ biotic pollination, $\mathrm{C} 4=\mathrm{C} 4$ or $\mathrm{CAM}$ photosynthetic pathway, $\mathrm{NF}=$ nitrogen fixer, $\mathrm{MY}=$ mycorrhizae, $\mathrm{ON}=$ other nutrient uptake strategy, $\mathrm{TU}=$ tussock, $\mathrm{WO}=$ woody, $\mathrm{SU}=$ succulent, $\mathrm{EP}=$ epiphyte, $\mathrm{CL}=$ climber, $\mathrm{AQ}=$ aquatic, $\mathrm{PA}=$ parasite, $\mathrm{OG}=$ other growth form, $\mathrm{CA}=$ clonal aboveground, $\mathrm{CB}=$ clonal belowground, $\mathrm{WI}=$ wind dispersal, $\mathrm{AI}=$ animal dispersal (consumption), $\mathrm{AE}=$ animal dispersal (external), $\mathrm{WA}=$ water dispersal.

altered disturbance regimes and deliberate introduction of plants with traits that appeal to humans (e.g. fast growth rates, showy flowers).

\section{Traits influence colonization and extirpation}

Consistent with previous work (Thompson and McCarthy 2008), our results point to seed mass and plant height as key traits influencing species success in urban floras (Fig. 6b). They are consistently involved in extirpation and colonization in urban areas, a result also reported in a synthesis of the urban plant trait literature by Williams et al. (2015). Colonizers and extirpated plants have previously been hypothesised to occupy opposite extremes of the trait spectrum, with persistent plants pooled at some middle point between those extremes (McKinney and Lockwood 1999); a theory that has received some support from studies of the Fabaceae family at global level (Bradshaw et al. 2008) and riparian communities in Australia (Kyle and Leishman 2009). Although we found similar patterns for seed mass and plant height, none of the remaining traits included in the study supported this hypothesis. In regards to seed mass and plant height, our results suggest that colonizers in urban areas are not only mimicking the strategies used by persistent plants, but they further show more extreme trait values that may confer the ability to fill novel niche space created through urbanization, shifting the community functional composition towards taller and heavier-seeded species. Because these traits are implicated in extirpation as well as colonization, these patterns do not appear to arise from an introduction bias of colonizers (Colautti et al. 2006), but rather suggest a causal link between these traits and success in urban environments.
Our work suggests that short life-history and unspecialised nutrient acquisition strategies promote colonization, but do not influence extirpation, in urban areas (Fig. 6d). In line with our results, Pyšek and Richardson (2007) found that naturalized species have different (though not necessarily shorter) longevity than the communities they colonize. Given the high levels of nutrients in urban soils, specialised nutrient acquisition mechanisms, such as nitrogen fixation, would offer little advantage and may even be detrimental if they involve an unnecessary investment of energy that otherwise could be used to increase individual fitness. We found some uncertainty on the effects of nutrient acquisition strategies, which is likely related to the high prevalence of plant-mycorrhiza associations among vascular plants in comparison to other strategies, regardless of being colonizers (Bunn et al. 2015). Finally, our results suggest that wind dispersal does not promote colonization, which contradicts previous work reporting greater frequency of wind dispersal for naturalized plants (Lake and Leishman 2004, Gassó et al. 2009). This contradiction may be explained by the fact that we examined the effect of wind dispersal versus no specialised dispersal features (Table 1), and not wind dispersal versus specialised dispersal vectors other than wind.

Environmental changes from urbanization appear to have altered the types of species that can persist in these areas (Fig. 6b, d), thereby shifting the functional composition of local plant communities toward species with one or more of the following traits: tall stature, heavy seeds, short lifehistory, and unspecialised nutrient uptake strategies and dispersal vector. Our results suggest that plants rely on different ecological strategies and mechanisms to occupy urban environments: plants taller than their neighbours have a competitive advantage through preferential light capture 
(a)
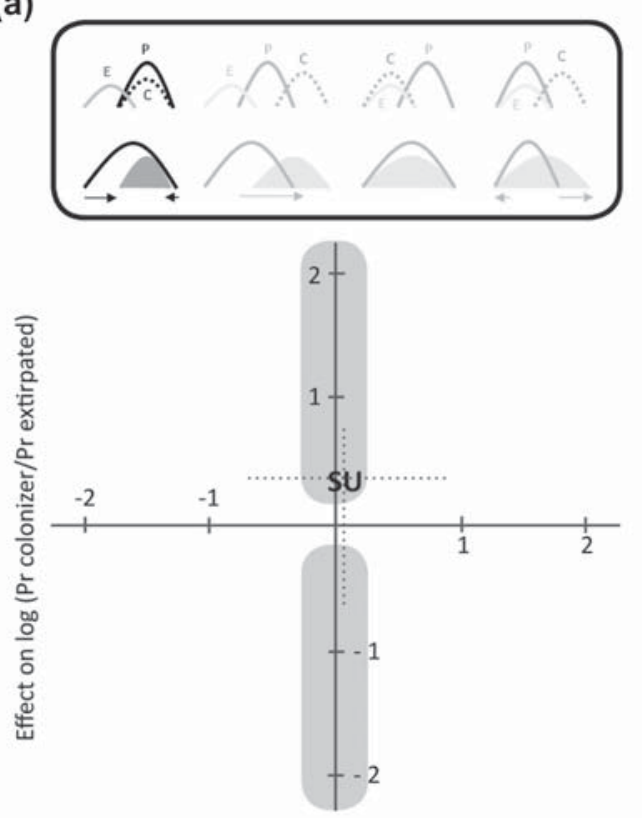

Effect on log ( $\mathrm{Pr}$ colonizer/Pr persistent)

(c)

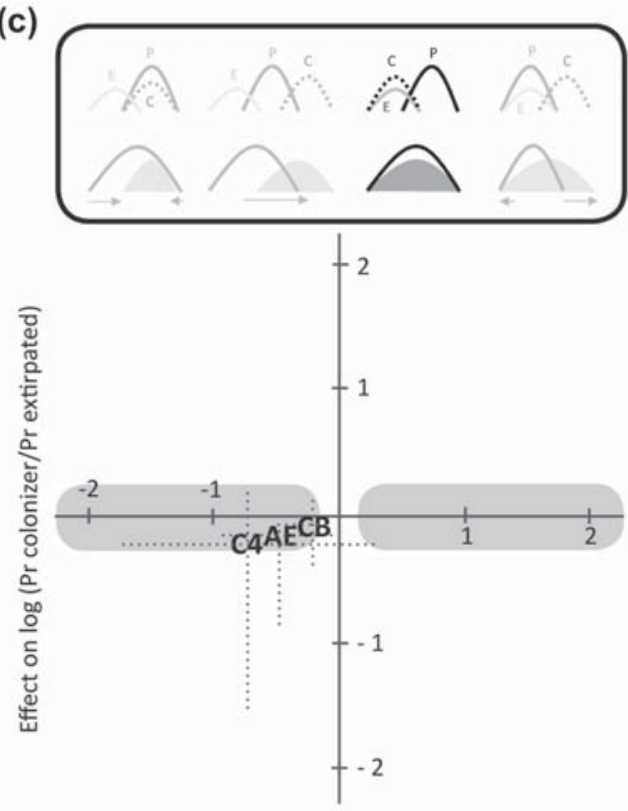

Effect on log ( $\mathrm{Pr}$ colonizer/Pr persistent) (b)
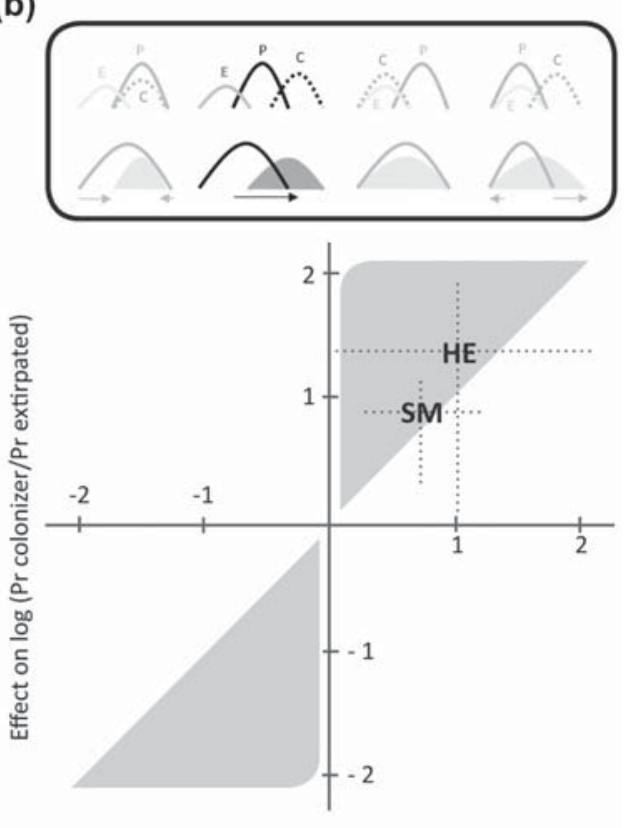

Effect on $\log (\operatorname{Pr}$ colonizer/Pr persistent)

(d)

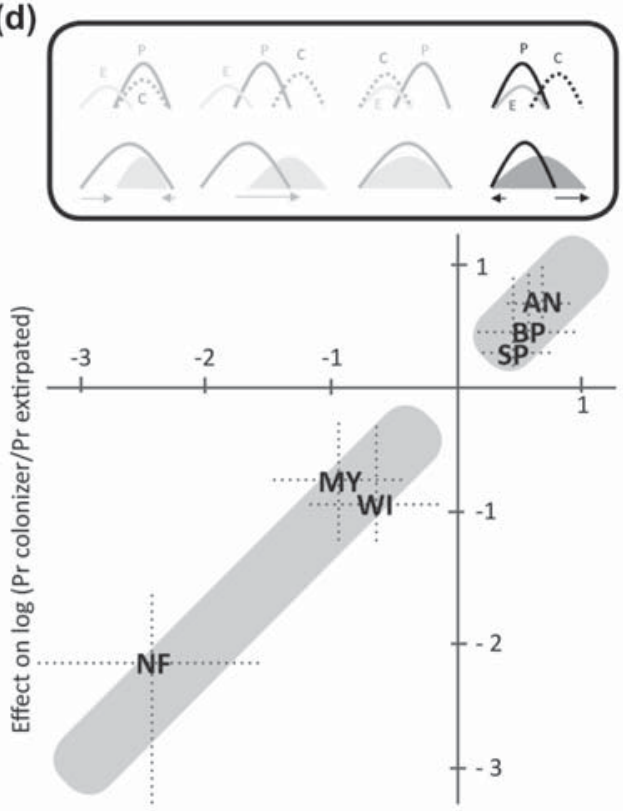

Effect on $\log (\mathrm{Pr}$ colonizer/Pr persistent)

Figure 6. Estimated effect of traits on the log odds of colonizers over persistent plants (x axis) and estimated effect of traits on the log odds of colonizers over extirpated plants (y axis), based on effects estimated from the meta-analyses. Trait codes represent mean effects and dashed lines represent $95 \%$ credible interval. Subplots represent different scenarios of functional change, based on the inferred distributions of trait values, following Fig. 1; the community-level trait distribution narrows (a), shifts (b), remains (c), or becomes wider (d). Traits not displayed in the figure did not show any consistent pattern concerning our hypotheses of functional change (i.e. laid outside the plot space of interest, shaded in grey for each scenario). Codes for traits: $\mathrm{SM}=$ seed mass, $\mathrm{HE}=$ height, $\mathrm{AN}=$ annual longevity, $\mathrm{SP}=$ spines, $\mathrm{BP}=$ biotic pollination, $\mathrm{C} 4=\mathrm{C} 4$ or $\mathrm{CAM}$ photosynthetic pathway, $\mathrm{NF}=$ nitrogen fixer, $\mathrm{MY}=$ mycorrhizae, $\mathrm{SU}=$ succulent, $\mathrm{CB}=$ clonal belowground, $\mathrm{WI}=$ wind dispersal.

and further dispersal (Westoby et al. 2002); heavier seeds have shown higher probability of establishment under harsh conditions (e.g. light or water shortage; Moles and Westoby (2002)); a short life-cycle allows fast growth rates and colonisation of disturbed patches with high resource availability; unspecialised nutrient and dispersal strategies allow plants to save the energetic investment of specialised organs, which may be used for other purposes (e.g. reproduction). This paper focuses on plant traits associated with success in urban environments rather than traits linked with the invasiveness 
of non-native species. Nonetheless, there seem to be some commonalities between the two, suggesting that similar processes and plant adaptations are important. Research in invasion ecology has shown that success of invasive, compared to non-invasive, non-native plants is also related to individual size (Van Kleunen et al. 2010), seed mass, clonal ability (Lake and Leishman 2004) and high rates of nutrient uptake under elevated nutrient concentrations (Matzek 2012).

We also found that spinescence and animal-driven pollination promote colonization, but do not affect extirpation. These patterns may result from a biased introduction of plants due to human preferences towards species with particular traits (Knapp et al. 2010), or in the case of spiny plants, due to lower probability of trampling or hand removal after establishment.

\section{Replacement of extirpated urban plants}

For some traits, we found that colonizers are more similar to extirpated than persistent urban plants (Fig. 6c), a fact that may be understood as colonizers replacing functionally similar extirpated species (Fig. 1c). Some of these findings were unexpected. For example, clonality has been previously reported to be higher in naturalized species in Australia and several European countries (Thompson et al. 1995), which may result from a climatic mismatch affecting the production of viable seeds and sexual reproduction.

The fact that colonizers and extirpated urban species share functional characteristics points to little or no change on some aspects of the functional composition of the urban plant community regardless of taxonomic changes in urban floras. Whether this replacement results from competition (based on traits different than resource acquisition strategy) or is a consequence of introduction of new species after removal of previously established plants remains to be investigated.

\section{Differences among cities}

The influence of local characteristics of urban areas (e.g. initial plant community composition and functional diversity) became obvious when effects of traits were compared across city-level analyses (Olden and Poff 2003). Cities belonging to Oceania (Adelaide, Melbourne and Auckland) and tropical Asian cities (Hong Kong and Singapore) showed clustered and, in some cases, extreme main effects for some traits (Supplementary material Appendix 1, Table A4 and A5). One example is the diverse city-level effects of seed mass in the comparison between colonizers and persistent species (Supplementary material Appendix 1, Fig. A2a). The strength of the relationship between this trait and colonizers varies between clusters of Asian, North American and Oceanian cities. High seed mass seems to be a distinctive characteristic of colonizers in Adelaide, Melbourne and Auckland. However, in Singapore and Hong Kong, colonizers and persistent species do not show differences in seed mass. As the latter are tropical cities, it is possible that their original plant community comprises species with heavier seeds than those in temperate regions, such as North America and Oceania (Moles et al. (2007); but see Chicago, where colonizer and persistent plants show similar seed mass values). The variability among cities may partially reflect the different developmental histories of the studied cities and the varying definition of city boundaries - and, therefore, local plant communities - as different parts of the landscape (e.g. highly urbanized patch vs vegetation remanent) support different assemblages of traits. The importance of regional differences was reinforced by our exploration of sources of variability on trait effects (Fig. 5). With only 11 cities, we did not have sufficient power to determine precise relationships between traits and extirpation/colonization across different regions of the world; such an exploration would require a larger number of cities per biogeographic region and was thus beyond the scope of this paper. Determining how biogeography, climate and urban history may modulate the functional composition of urban floras presents an exciting opportunity for future research. By accounting for regional variation, a hierarchical investigation of both region- and city-level trait effects in urban floras would likely reduce uncertainty in estimated relationships. As such, our estimations of global effects presented here should be understood as conservative quantifications, since the uncertainty associated with them still allows some variability between regions and cities.

\section{Caveats of this work}

Multiple processes determine the types of species able to occupy urban environments: human preferences and species dispersal opportunities will influence the types of species able to colonize cities, while human choices about the types and locations of remaining vegetation within cities will partially shape extirpation patterns. Positive/ negative effects of traits do not imply that they affect species' success at every step, or consistently across the landscape. We are aware of the potential limitations of working with unstandardized city boundaries since cities including nearby natural areas (e.g. San Diego) may potentially show lower rates of extirpation. However, Hahs et al. (2009) found that development history and the extent of original vegetation remaining within 22 cities were important determinants of plant extirpation rates. We acknowledge that phylogenetic relatedness could have been accounted for using different approaches. We decided to use family as a surrogate of relatedness because it provided a balanced allocation of trait variations between evolution and present-day ecology (Westoby 1998). While our approach enables correct estimation of the effects of interest, it may poorly predict estimates using species with different phylogenetic relationships. The stronger relationship we found between traits and colonization rather than extirpation may be an artefact of the specific set of traits we included in the study, so findings of studies that include a different set of traits may differ. In addition, the high stochasticity associated with a small sample size (i.e. 11 cities) could have prevented us from distinguishing general relationships between traits and extirpation and colonization processes from local patterns (Niemelä et al. 2009).

In this work we present average effects of traits (not predictions of trait distributions), we assume that benefits 
gained from having particular traits are consistent through time, and we do not consider local adaptation or evolution in urban environments as possible mechanisms contributing to local plant survival. There is a chance our data set contains some misclassifications, in the form of false persistents (i.e. plants on the path to extirpation, but still remaining in small populations; the extinction debt, Tilman et al. (1994)), and false colonizers (i.e. persistent plants that went undetected by early surveys). The extinction debt may explain why we found woody growth forms to be negatively associated with extirpation (Fig. 4), when other studies have found that many urban colonizers are long-lived woody plants (Williams et al. 2015). The presence of false colonizers would bias our inference only for traits correlated with an increased probability of plant detection, such as height (Garrard et al. 2013). Since we found colonizers were in fact the tallest plants, we are confident colonizer misclassifications did not affect our results. Finally, we did not consider the formal inclusion of the spatial and temporal grain of the plant surveys in our analyses. A simple exploration of their effects on the rates of extirpation and colonization of our 11 cities showed that, in our case, these elements are not likely to affect our results (Supplementary material Appendix 2), although they may influence the biotic change output in other studies (Olden and Poff 2003).

\section{Conclusion}

In this paper, we investigated whether urban environments consistently select for particular types of plant traits and investigated the implications for the functional composition of the flora of 11 cities. We investigated these patterns in the same cities where Duncan et al. (2011) found that seed mass and height can be used to characterize extirpation-prone (vs persisting) plants in urban environments. In the work presented here, we examined the characteristics of plants that are both lost and gained from urban environments, which allows exploration of different scenarios of biotic change (Olden and Poff 2003). Our results showed that, in addition to seed mass and height, which are driving a shift in urban floras via local extirpation and colonization, other traits such as longevity are also driving some consistent changes in urban floras' functional composition. Understanding the consequences of biotic changes requires a simultaneous assessment of extirpated and colonizing species; our study investigated the relationship between plant traits and both extirpation and colonization processes to provide a clear picture of the several possibilities of functional change occurring in human-dominated habitats.

Acknowledgements - The database used for this analysis was initially compiled by the 'Plant traits and urbanization' working group funded by the ARC-NZ Network for Vegetation Function. EP would like to thank Maud Bernard-Verdier for stimulating conversations on functional change. EP was supported by a Melbourne International Research Scholarship and a Melbourne International Fee Remission Scholarship, granted by the Univ. of Melbourne. JAC, MAM and PAV were supported by the ARC Centre of Excellence for Environmental Decisions. AKH and MJM would like to acknowledge funding from the Baker Foundation and JAC from the ARC (DE120102221).

\section{References}

Angiosperm Phylogeny Group 2003. An update of the Angiosperm Phylogeny Group classification for the orders and families of flowering plants: APG II. - Bot. J. Linn. Soc. 141: 399-436.

Arnqvist, G. and Wooster, D. 1995. Meta-analysis: synthesizing research findings in ecology and evolution. - Trends Ecol. Evol. 10: 236-240.

Bradshaw, C. J. A. et al. 2008. Threat or invasive status in legumes is related to opposite extremes of the same ecological and lifehistory attributes. - J. Ecol. 96: 869-883.

Bunn, R. A. et al. 2015. Do native and invasive plants differ in their interactions with arbuscular mycorrhizal fungi? A metaanalysis. - J. Ecol. 103: 1547-1556.

Cohen, J. E. 2003. Human population: the next half century. - Science 302: 1172-1175.

Colautti, R. I. et al. 2006. Propagule pressure: a null model for biological invasions. - Biol. Invasions 8: 1023-1037.

Cornelissen, J. H. C. et al. 2003. A handbook of protocols for standardised and easy measurement of plant functional traits worldwide. - Aust. J. Bot. 51: 335-380.

Díaz, S. et al. 2004. The plant traits that drive ecosystems: evidence from three continents. - J. Veg. Sci. 15: 295-304.

Duncan, R. P. and Young, J. R. 2000. Determinants of plant extinction and rarity 145 years after European settlement of Auckland, New Zealand. - Ecology 81: 3048-3061.

Duncan, R. P. et al. 2011. Plant traits and extinction in urban areas: a meta-analysis of 11 cities. - Global Ecol. Biogeogr. 20: 509-519.

Gallagher, R. V. et al. 2013. Species loss and gain in communities under future climate change: consequences for functional diversity. - Ecography 36: 531-540.

Garrard, G. E. et al. 2013. A general model of detectability using species traits. - Methods Ecol. Evol. 4: 45-52.

Gassó, N. et al. 2009. Exploring species attributes and site characteristics to assess plant invasions in Spain. - Divers. Distrib. 15: $50-58$.

Gelman, A. and Hill, J. 2007. Data analysis using regression and multilevel/hierarchical models. - Cambridge Univ. Press.

Grimm, N. B. et al. 2008. Global change and the ecology of cities. - Science 319: 756-760.

Hahs, A. K. et al. 2009. A global synthesis of plant extinction rates in urban areas. - Ecol. Lett. 12: 1165-1173.

Hodkinson, D. J. and Thompson, K. 1997. Plant dispersal: the role of man. - J. Appl. Ecol. 34: 1484-1496.

Jeschke, J. M. and Strayer, D. L. 2008. Are threat status and invasion success two sides of the same coin? - Ecography 31: 124-130.

Keddy, P. A. 1992. Assembly and response rules: two goals for predictive community ecology. - J. Veg. Sci. 3: 157-164.

Knapp, S. et al. 2010. Changes in the functional composition of a central European urban flora over three centuries. - Perspect. Plant Ecol. Evol. Syst. 12: 235-244.

Koricheva, J. et al. 2013. Handbook of meta-analysis in ecology and evolution. - Princeton Univ. Press.

Kyle, G. and Leishman, M. R. 2009. Functional trait differences between extant exotic, native and extinct native plants in the Hunter River, NSW: a potential tool in riparian rehabilitation. - River Res. Appl. 25: 892-903.

La Sorte, F. A. et al. 2014. Beta diversity of urban floras among European and non-European cities. - Global Ecol. Biogeogr. 23: 769-779.

Lake, J. C. and Leishman, M. R. 2004. Invasion success of exotic plants in natural ecosystems: the role of disturbance, plant attributes and freedom from herbivores. - Biol. Conserv. 117: 215-226.

Lavorel, S. et al. 2007. Plant functional types: are we getting any closer to the Holy grail? - In: Canadell, J. et al. (eds), Terrestrial ecosystems in a changing world. Springer, pp. 149-164. 
Leach, M. K. and Givnish, T. J. 1996. Ecological determinants of species loss in remnant prairies. - Science 273: 1555-1558.

Matzek, V. 2012. Trait values, not trait plasticity, best explain invasive species' performance in a changing environment. - PLoS One 7: e48821.

McDonnell, M. J. and Hahs, A. K. 2015. Adaptation and adaptedness of organisms to urban environments. - Annu. Rev. Ecol. Evol. Syst. 46: 261-280.

McGill, B. J. et al. 2006. Rebuilding community ecology from functional traits. - Trends Ecol. Evol. 21: 178-185.

McKinney, M. L. 2002. Urbanization, biodiversity, and conservation. - Bioscience 52: 883-890.

McKinney, M. L. and Lockwood, J. L. 1999. Biotic homogenization: a few winners replacing many losers in the next mass extinction. - Trends Ecol. Evol. 14: 450-453.

Moles, A. T. and Westoby, M. 2002. Seed addition experiments are more likely to increase recruitment in larger-seeded species. - Oikos 99: 241-248.

Moles, A. T. et al. 2007. Global patterns in seed size. - Global Ecol. Biogeogr. 16: 109-116.

Niemelä, J. et al. 2009. Comparative urban ecology: challenges and possibilities. - In: McDonnell, M. J. et al. (eds), Ecology of cities and towns: a comparative approach. Cambridge Univ. Press, pp. 9-24.

Olden, J. D. 2006. Biotic homogenization: a new research agenda for conservation biogeography. - J. Biogeogr. 33: 2027-2039.

Olden, J. D. and Poff, N. L. 2003. Toward a mechanistic understanding and prediction of biotic homogenization. - Am. Nat. 162: 442-460.

Olden, J. D. and Rooney, T. P. 2006. On defining and quantifying biotic homogenization. - Global Ecol. Biogeogr. 15: 113-120.

Ozinga, W. A. et al. 2009. Dispersal failure contributes to plant losses in NW Europe. - Ecol. Lett. 12: 66-74.

Pyšek, P. and Richardson, D. M. 2007. Traits associated with invasiveness in alien plants: where do we stand? - In: Nentwig, W. (ed.), Biological invasions. Springer, pp. 97-125.

Rejmánek, M. 2011. Invasiveness. - In: Simberloff, D. and Rejmanek, M. (eds), Encyclopedia of biological invasions. Univ. of California Press, pp. 379-385.

Supplementary material (Appendix ECOG-02516 at $<$ www.ecography.org/appendix/ecog-02516>). Appendix 1-3.
Richardson, D. M. et al. 2000. Naturalization and invasion of alien plants: concepts and definitions. - Divers. Distrib. 6: 93-107.

Shochat, E. et al. 2006. From patterns to emerging processes in mechanistic urban ecology. - Trends Ecol. Evol. 21: 186-191.

Sodhi, N. S. et al. 2008. Correlates of extinction proneness in tropical angiosperms. - Divers. Distrib. 14: 1-10.

Sutherland, S. 2004. What makes a weed a weed: life history traits of native and exotic plants in the USA. - Oecologia 141: 24-39.

Thompson, K. and McCarthy, M. A. 2008. Traits of British alien and native urban plants. - J. Ecol. 96: 853-859.

Thompson, K. and Davis, M. A. 2011. Why research on traits of invasive plants tells us very little. - Trends Ecol. Evol. 26: 155-156.

Thompson, K. et al. 1995. Native and alien invasive plants: more of the same? - Ecography 18: 390-402.

Tilman, D. et al. 1994. Habitat destruction and the extinction debt. - Nature 371: 65-66.

Van Kleunen, M. et al. 2010. A meta-analysis of trait differences between invasive and non-invasive plant species. - Ecol. Lett. 13: $235-245$.

Vitousek, P. M. et al. 1997. Human domination of Earth's ecosystems. - Science 277: 494-499.

Westoby, M. 1998. Generalization in functional plant ecology: the species sampling problem, plant ecological strategy schemes, and phylogeny. - In: Pugnaire, F. and Valladares, F. (eds), Handbook of functional plant ecology. Marcel Dekker, pp. 685-703.

Westoby, M. et al. 2002. Plant ecological strategies: some leading dimensions of variation between species. - Annu. Rev. Ecol. Syst. 33: 125-159.

Williams, N. S. G. et al. 2005. Plant traits and local extinctions in natural grasslands along an urban-rural gradient. - J. Ecol. 93: 1203-1213.

Williams, N. S. G. et al. 2009. A conceptual framework for predicting the effects of urban environments on floras. - J. Ecol. 97: 4-9.

Williams, N. S. G. et al. 2015. Urbanisation, plant traits and the composition of urban floras. - Perspect. Plant Ecol. Evol. Syst. 17: 78-86. 